\title{
Optimal Resource Allocation for Energy-Efficient OFDMA Networks
}

\author{
Fan Wu, Yuming Mao, Xiaoyan Huang, and Supeng Leng \\ Key Laboratory of Optical Fiber Sensing and Communications, School of Communication and Information Engineering, \\ University of Electronic Science and Technology of China, Chengdu 611731, China \\ Correspondence should be addressed to Supeng Leng; spleng@uestc.edu.cn
}

Received 22 September 2014; Revised 27 February 2015; Accepted 1 March 2015

Academic Editor: Alex Elías-Zúñiga

Copyright (C) 2015 Fan Wu et al. This is an open access article distributed under the Creative Commons Attribution License, which permits unrestricted use, distribution, and reproduction in any medium, provided the original work is properly cited.

\begin{abstract}
This paper focuses on radio resource allocation in OFDMA networks for maximizing the energy efficiency subject to the data rate requirements of users. We propose the energy-efficient water-filling structure to obtain the closed-form optimal energy-efficient power allocation for a given subcarrier assignment. Moreover, we establish a new sufficient condition for the optimal energyefficient subcarrier assignment. Based on the theoretical analysis, we develop a joint energy-efficient resource allocation (JERA) algorithm to maximize the energy efficiency. Simulation results show that the JERA algorithm can yield optimal solution with significantly low computational complexity.
\end{abstract}

\section{Introduction}

As the high data rate applications are going to dominate the mobile services, energy efficiency (EE) is becoming more crucial in wireless communication networks. Energy-efficient radio resource allocation is one of the effective ways to improve the EE of the OFDMA (orthogonal frequency division multiple access) networks [1]. Although radio resource allocation in OFDMA networks has been extensively studied, the major focus is on improving spectral efficiency (SE) which may not always coincide with EE [2].

Different from SE-based resource allocation schemes in which the total transmitting power is fixed, the EEbased schemes adjust the power level adaptively based on the channel conditions [3]. Accordingly, the classic waterfilling power allocation method cannot be applied directly due to the unknown total transmitting power. In order to determine the proper transmitting power, the perturbation functions of EE have been studied in $[4,5]$. It has been shown that EE is strictly quasi-concave in SE [4] and in the total transmitting power [5]. However, the perturbation functions of EE cannot be expressed in an analytic expression; only the approximation algorithms have been proposed to find the near-optimal solutions [4-6].

In this paper, we focus on joint subcarrier assignment and power allocation in OFDMA networks for maximizing the energy efficiency problem subject to the data rate requirements of users. The main contributions of our work are summarized as follows.

(i) We prove that EE is strictly pseudo-concave with respect to power vector for a given subcarrier allocation, which guarantees that the solution satisfying the KKT conditions is also the global optimal. Using this property, we show that the optimal solution has a special EE water-filling structure that is determined by only one variable. Based on this observation, we further provide the first closed-form expression for the optimal energy-efficient power allocation.

(ii) According to the analysis, we propose an optimal energy-efficient power allocation algorithm by sequentially searching within a finite number of water-level intervals. The computational complexity of the proposed algorithm is much lower than that of 
the algorithms approaching the optimal solution with iterative searching method.

(iii) We provide a sufficient condition for the optimal energy-efficient subcarrier assignment. This condition is the basis of the quick search method, because it allows us to easily determine whether a subcarrier assignment is optimal. As a result, by combining the sufficient condition and the EE water-filling solution, we design a joint energy-efficient resource allocation (JERA) algorithm, which not only achieves the optimal EE, but also outperforms the existing algorithms in terms of convergence rate.

The state of the art to solve the EE maximization problem is JIOO (joint inner- and outer-layer optimization) algorithm $[4,5]$. Relying on the strict quasi-concavity of the perturbation function of EE with respect to the total transmitting power, the JIOO algorithm solves the problem via two-layer iterations, where the inner layer aims to find the maximum $\mathrm{EE}$ and its derivative for a given total transmitting power, and the outer layer targets to search for the total transmitting power which results in the maximum EE by bisection search. The computational complexity is mainly determined by the outer layer iterations. Unfortunately, the number of the outer layer iterations becomes infinite when tolerance approaches zero. Different from this approach, the complexity of our algorithm depends on the finite number of the feasible subcarrier assignments. Moreover, the search space can be greatly reduced by using the sufficient condition provided by this paper. In addition, the EE water-filling structure ensures that the results obtained by our algorithm are the exact optimal solutions other than approximation ones.

The rest of the paper is organized as follows. In Section 2, we describe the system model and formulate the EE maximization problem for the downlink OFDMA networks. The optimal energy-efficient power allocation for a given subcarrier assignment and the sufficient condition for the optimal energy-efficient subcarrier assignment are studied in Sections 3 and 4, respectively. An optimal energy-efficient power allocation algorithm and a low-complexity joint resource allocation algorithm are elaborated in Section 5. Section 6 provides the performance evaluation of the proposed algorithms via simulations. Finally, we conclude this paper in Section 7.

\section{System Model}

Consider a downlink OFDMA network with one base station and $K$ users. Let $\mathscr{K}=\{1, \ldots, K\}$ and $\mathscr{N}=\{1, \ldots, N\}$ be the set of users and subcarriers, respectively. Define the subcarrier assignment matrix $\boldsymbol{\rho}=\left(\rho_{k, n}\right)_{K \times N}$ where $\rho_{k, n}=1$ means that subcarrier $n$ is allocated to user $k$, and otherwise $\rho_{k, n}=0$. The transmitting power allocation matrix is defined as $\mathbf{p}=\left(p_{k, n}\right)_{K \times N}$, where $p_{k, n} \geq 0$ represents the transmitting power allocated to user $k$ on subcarrier $n$. Then, the maximum achievable data rate of user $k$ on subcarrier $n$ is given by

$$
r_{k, n}=B \log _{2}\left(1+p_{k, n} \gamma_{k, n}\right)
$$

where $B$ is the bandwidth of subcarrier and $\gamma_{k, n}$ denotes the normalized channel power gain of user $k$ on subcarrier $n$. Accordingly, the overall system data rate is

$$
R=\sum_{k=1}^{K} \sum_{n=1}^{N} r_{k, n} \cdot \rho_{k, n},
$$

and the total transmitting power is

$$
P_{T}=\sum_{k=1}^{K} \sum_{n=1}^{N} p_{k, n} \cdot \rho_{k, n} .
$$

In addition to transmitting power, the energy consumption also includes circuit power which is consumed by device electronics. The circuit power is modeled as a constant $P_{C}$, which is independent of data transmission rate [7]. Accordingly, we define EE as the amount of bits transmitted per Joule of energy; that is, $\eta_{\mathrm{EE}} \triangleq R /\left(\zeta P_{T}+P_{C}\right)$, where $\zeta$ is the reciprocal of drain efficiency of power amplifier.

In our work, we consider maximizing EE under the minimum data rate requirements, $R_{k}$, and the total transmitting power constraint, $P_{\max }$. Accordingly, this optimization problem can be formulated as

$$
\begin{aligned}
\mathrm{P} 1: & \max _{\mathbf{p}, \boldsymbol{\rho}} \frac{\sum_{k=1}^{K} \sum_{n=1}^{N} r_{k, n} \cdot \rho_{k, n}}{\zeta P_{T}+P_{C}} \\
\text { s.t. } & \sum_{n=1}^{N} r_{k, n} \cdot \rho_{k, n} \geq R_{k}, \quad \forall k \in \mathscr{K}, \\
& P_{T} \triangleq \sum_{k=1}^{K} \sum_{n=1}^{N} p_{k, n} \cdot \rho_{k, n} \leq P_{\max }, \\
& \sum_{k=1}^{K} \rho_{k, n}=1, \quad \forall n \in \mathscr{N}, \\
& \rho_{k, n} \in\{0,1\}, \quad p_{k, n} \geq 0, \quad \forall k \in \mathscr{K}, \quad \forall n \in \mathscr{N},
\end{aligned}
$$

where $(4 \mathrm{~b})$ indicates the minimum data rate requirement of each user, (4c) is the total transmitting power constraint, and (4d) is the constraint on subcarrier assignment to ensure that each subcarrier is only assigned to one user.

Similar to the traditional spectral-efficient resource model, $\mathrm{P} 1$ is a mixed integer nonlinear programming problem, and it is not trivial to obtain the global optimal solution to this problem. To solve the problem, we first decompose P1 into two subproblems, which include (1) the energy-efficient power allocation for a fixed subcarrier assignment $\boldsymbol{\rho}$ and (2) the energy-efficient subcarrier assignment for a given total transmitting power $P_{T}$. Then, based on the properties of the subproblems, we develop an algorithm to find the solution of joint energy-efficient power allocation and subcarrier assignment to maximize EE.

\section{Optimal Energy-Efficient Power Allocation}

In this section, we analyze the optimal energy-efficient power allocation (EPA) based on the EE water-filling structure. All 
the major results are given by some theorems. In particular, Theorems 2 and 5 demonstrate that the global optimal solution to the energy-efficient power optimization problem is with the EE water-filling structure. Theorem 3 provides the corresponding closed-form water-level, whose optimality is proved by Theorem 4 .

3.1. EE Water-Filling Structure. Given the subcarrier assignment matrix $\rho$, the set of subcarriers assigned to user $k$ can be denoted by $\mathscr{N}^{\boldsymbol{\rho}}(k)=\left\{n \mid \rho_{k, n}=1\right\}$ and the power vector $\mathbf{p}=\left(p_{1} \cdots p_{n}\right)^{T}$. Then P1 is reduced to the following EPA problem:

$$
\begin{gathered}
\mathrm{P} 2: \quad \eta_{\mathrm{EE}}^{(\boldsymbol{\rho})} \triangleq \max _{\mathbf{p} \geq 0} \eta_{\mathrm{EE}}^{(\boldsymbol{\rho})}(\mathbf{p}) \\
\text { s.t. } r_{k}(\mathbf{p}) \geq R_{k} \quad \forall k \in \mathscr{K}, \\
p_{T}(\mathbf{p}) \leq P_{\max },
\end{gathered}
$$

where $\eta_{\mathrm{EE}}^{(\boldsymbol{\rho})}(\mathbf{p}) \triangleq \sum_{n=1}^{N}\left(B \log _{2}\left(1+\gamma_{n} p_{n}\right) /\left(\zeta \sum_{n=1}^{N} p_{n}+P_{C}\right)\right)$ with $\gamma_{n}=\gamma_{k(n), n}$, and $k(n)$ denotes the index of the user assigned on subcarrier $n \cdot r_{k}(\mathbf{p}) \triangleq \sum_{n \in \mathcal{N}^{\rho}(k)} B \log _{2}\left(1+\gamma_{n} p_{n}\right)$, and $p_{T}(\mathbf{p}) \triangleq \sum_{n=1}^{N} p_{n}$. Since the numerator of $\eta_{\mathrm{EE}}^{(\boldsymbol{\rho})}(\mathbf{p})$ is differentiable, positive, and strictly concave function in $\mathbf{p}$ and the denominator is positive and affine in $\mathbf{p}, \eta_{\mathrm{EE}}^{(\rho)}(\mathbf{p})$ is a strictly pseudo-concave function with respect to $\mathbf{p}$ [8]. Besides, $r_{k}(\mathbf{p})$ is differentiable and concave for all $k$, and $p_{T}(\mathbf{p})$ is positive and affine. Therefore, according to the KKT sufficient optimality theorem [9], any feasible solution $\mathbf{p}$ satisfying the KKT conditions is also globally optimal for P2.

When the feasible solution set of P2 is nonempty, the minimum power vector $\check{\mathbf{p}}$ to guarantee the minimum datarate requirement of each user must be a feasible solution to $\mathrm{P} 2$, which can be obtained by solving the following margin adaptive (MA) problem [10]:

$$
\begin{aligned}
& \text { P3: } P_{\min } \triangleq \min _{p_{n} \geq 0} \sum_{n=1}^{N} p_{n} \\
& \text { s.t. } r_{k}(\mathbf{p})=R_{k}, \quad \forall k \in \mathscr{K} .
\end{aligned}
$$

Resorting to the Lagrange dual theory, the optimal solution to $\mathrm{P} 3$ is given by $\check{\mathbf{p}}=\left(\left(\check{x}_{k(n)}-1 / \gamma_{n}\right)^{+}\right)_{N \times 1}$, where $\check{x}_{k}$ is the root of the equation

$$
\sum_{n \in \mathscr{N}^{\boldsymbol{\rho}}(k)} B \log _{2}\left[1+\gamma_{n}\left(\check{x}_{k}-\frac{1}{\gamma_{n}}\right)^{+}\right]=R_{k}, \quad \forall k \in \mathscr{K} .
$$

We call $\check{x}_{k}$ the lowest power water-level of user $k$.

More importantly, a series of feasible solutions to P2 can be constructed based on $\check{\mathbf{p}}$ by raising the water-levels of some users and maintaining that of the others. To be specific, by sorting all the users in ascending order of their lowest power water-levels such that $\check{x}_{1} \leq \cdots \leq \check{x}_{K+1}$ with $\check{x}_{K+1}=+\infty$, the region of the promotable water-level can be divided into $K$ intervals, that is, $\left(\check{x}_{1}, \check{x}_{2}\right], \ldots,\left(\check{x}_{K}, \check{x}_{K+1}\right]$, named as waterlevel rise interval. For the lth $(0<l \leq K)$ interval, given $x \in$ $\left(\check{x}_{l}, \check{x}_{l+1}\right]$ called EE water-level, if we raise the water-levels of the first $l$ users to $x$, while maintaining that of other $K-l$ users, a new feasible power solution $\check{\mathbf{p}}(x)$ can be obtained by the following EE water-filling structure:

$$
\check{p}_{n}(x)= \begin{cases}\left(x-\frac{1}{\gamma_{n}}\right)^{+} & k \in \mathscr{K}_{1} \\ \left(\check{x}_{k(n)}-\frac{1}{\gamma_{n}}\right)^{+} & k \in \mathscr{K}_{2},\end{cases}
$$

where $\mathscr{K}_{1}=\{1 \cdots l\}$ and $\mathscr{K}_{2}=\mathscr{K}-\mathscr{K}_{1}$. The corresponding data rate of each user satisfies

$$
r_{k}(\check{\mathbf{p}}(x)) \begin{cases}>R_{k} & k(n) \in \mathscr{K}_{1} \\ =R_{k} & k(n) \in \mathscr{K}_{2} .\end{cases}
$$

It can be found that the data rate of each user with the same water-level is greater than the minimum requirement, while that of the other users is equal to the minimum requirement.

It is noteworthy that since $\check{x}_{k}$ in (8) can be obtained by solving (7) and hence $\check{\mathbf{p}}(x)$ can be expressed solely as a function of the water-level $x$, accordingly, P2 can be transformed into a single variable problem. Furthermore, for any given total transmitting power $P_{T}, \mathrm{P} 2$ is equivalent to $\mathrm{P} 4$ shown in the following. If the optimal solution to $\mathrm{P} 4$ has the EE water-filling structure, we can deduce that P2 must be maximized at a power vector with the EE water-filling structure. It can be proved by Theorem 1:

$$
\begin{aligned}
\text { P4: } & \max _{p_{n} \geq 0} R^{(\boldsymbol{\rho})}(\mathbf{p}) \\
\text { s.t. } & r_{k}(\mathbf{p}) \geq R_{k}, \quad \forall k \in \mathscr{K}, \\
& p_{T}(\mathbf{p})=P_{T},
\end{aligned}
$$

where $R^{(\boldsymbol{\rho})}(\mathbf{p}) \triangleq \sum_{n=1}^{N} B \log _{2}\left(1+\gamma_{n} p_{n}\right)$.

Theorem 1. Given $P_{T} \geq P_{\min }$, the optimal solution $\mathbf{p}^{*}$ to $P 4$ has the EE water-filling structure (8).

Proof. Since P4 is a convex programming, the optimal solution $\mathbf{p}^{*}$ must satisfy the KKT conditions; that is, there exist scalars $u_{k} \geq 0(k=1 \cdots K)$ and $\lambda$ such that

$$
\begin{gathered}
\nabla R^{(\boldsymbol{\rho})}\left(\mathbf{p}^{*}\right)+\sum_{k=1}^{K} u_{k} \nabla r_{k}\left(\mathbf{p}^{*}\right)+\lambda \nabla p_{T}\left(\mathbf{p}^{*}\right)=0, \\
u_{k} \cdot\left(r_{k}\left(\mathbf{p}^{*}\right)-R_{k}\right)=0, \quad k=1 \cdots K .
\end{gathered}
$$

According to (11), we have

$$
p_{n}^{*}=\left(B \cdot \frac{\left(1+u_{k(n)}\right)}{(\ln 2 \cdot \lambda)}-\frac{1}{\gamma_{n}}\right)^{+}, \quad \forall n
$$

Besides, based on the complementary slackness conditions (12), we can get that $u_{k}=0$ if $r_{k}\left(\mathbf{p}^{*}\right)>R_{k}$. Let $x \triangleq B /(\ln 2 \cdot \lambda)$ and $x_{k} \triangleq\left(1+u_{k(n)}\right) x$. Therefore, $\mathbf{p}^{*}$ can be further expressed as follows:

$$
\check{p}_{n}(x)= \begin{cases}\left(x-\frac{1}{\gamma_{n}}\right)^{+} & k(n) \in \mathscr{K}_{1}^{\prime} \\ \left(x_{k(n)}-\frac{1}{\gamma_{n}}\right)^{+} & k(n) \in \mathscr{K}_{2}^{\prime},\end{cases}
$$


where $\mathscr{K}_{1}^{\prime} \triangleq\left\{k \mid r_{k}\left(\mathbf{p}^{*}\right)>R_{k}\right\}$ and $\mathscr{K}_{2}^{\prime} \triangleq\left\{k \mid r_{k}\left(\mathbf{p}^{*}\right)=R_{k}\right\}$. To maximize the overall data rate, the power allocated to the users in $\mathscr{K}_{2}^{\prime}$ should be minimized. And then $x_{k}=\check{x}_{k}, \forall k \in$ $\mathscr{K}_{2}^{\prime}$. Since $u_{k} \geq 0$, we get $\check{x}_{k}=x_{k} \geq x$. On the other hand, $x>$ $\check{x}_{k}\left(\forall k \in \mathscr{K}_{1}^{\prime}\right)$ in order to satisfy $r_{k}\left(\mathbf{p}^{*}\right)>R_{k}$. Consequently, $\mathscr{K}_{1}^{\prime}=\mathscr{K}_{1}$ and $\mathscr{K}_{2}^{\prime}=\mathscr{K}_{2}$. We can now conclude that $\mathbf{p}^{*}$ has the EE water-filling structure as (8).

According to the water-levels of $\check{\mathbf{p}}(x)$, the subcarrier set can be further divided into three subsets: $\mathcal{N}_{0}=\left\{n \mid p_{n}=\right.$ $0, n \in \mathcal{N}\}, \mathcal{N}_{1}=\left\{n \mid p_{n}>0, k(n) \in \mathscr{K}_{1}\right\}$, and $\mathscr{N}_{2}=\{n \mid$ $\left.p_{n}>0, k(n) \in \mathscr{K}_{2}\right\}$. The partial derivatives of $\eta_{\mathrm{EE}}^{(\rho)}(\mathbf{p})$ have the following properties, which will be used in the proof of the following theorems.

Property 1. If $\check{\mathbf{p}}(x)$ is a feasible solution to $\mathrm{P} 2$, then

(a)

$$
\frac{\partial \eta_{\mathrm{EE}}^{(\boldsymbol{\rho})}(\check{\mathbf{p}}(x))}{\partial p_{n}}= \begin{cases}\frac{B /(\ln 2 \cdot x)-\zeta \eta_{\mathrm{EE}}^{(\boldsymbol{\rho})}(\check{\mathbf{p}}(x))}{\zeta P_{T}+P_{\mathrm{C}}} & n \in \mathcal{N}_{1} \\ \frac{B /\left(\ln 2 \cdot \check{x}_{k(n)}\right)-\zeta \eta_{\mathrm{EE}}^{(\boldsymbol{\rho})}(\check{\mathbf{p}}(x))}{\zeta P_{T}+P_{\mathrm{C}}} & n \in \mathcal{N}_{2} \\ \frac{B \gamma_{n} / \ln 2-\zeta \eta_{\mathrm{EE}}^{(\boldsymbol{\rho})}(\check{\mathbf{p}}(x))}{\zeta P_{T}+P_{\mathrm{C}}} & n \in \mathcal{N}_{0} ;\end{cases}
$$

(b)

$d_{1}(\check{\mathbf{p}}(x))=\cdots=d_{l}(\check{\mathbf{p}}(x)) \geq d_{l+1}(\check{\mathbf{p}}(x)) \geq \cdots \geq d_{K}(\check{\mathbf{p}}(x))$,

if $\check{x}_{l}<x \leq \check{x}_{l+1}$, where $d_{k}(\check{\mathbf{p}}(x)) \triangleq \max _{\forall n \in \mathcal{N}(k)} \partial \eta_{\mathrm{EE}}^{(\boldsymbol{\rho})}(\check{\mathbf{p}}(x)) /$ $\partial p_{n}$.

3.2. EE Water-Level Interval $[\check{x}, \widehat{x}]$. The minimum power vector $\check{\mathbf{p}}=\check{\mathbf{p}}(\check{x})$ is also with the EE water-filling structure whose water-level is $\check{x}_{1}$ and the total transmitting power is $P_{\min }$. On the other hand, suppose $\widehat{\mathbf{p}}$ is the optimal solution to $\mathrm{P} 4$ when $P_{T}=P_{\max }$; according to Theorem 1, we have $\widehat{\mathbf{p}}=$ $\check{\mathbf{p}}(\widehat{x})$, where $\widehat{x}$ is the EE water-level. Since the feasible region of $\mathrm{P} 1$ is nonempty, the total transmitting power $P_{T}$ must satisfy $P_{\min } \leq P_{T} \leq P_{\max }$. Hence, the corresponding power vector with the EE water-filling structure in the feasible region of P1 should be subject to $\check{\mathbf{p}}(x) \in \mathscr{P}=\{\check{\mathbf{p}}(x), x \in[\check{x}, \widehat{x}]\}$. Based on the strict pseudo-concavity of $\eta_{\mathrm{EE}}^{(\boldsymbol{\rho})}(\mathbf{p})$, we have the following theorem.

Theorem 2. Assume $\check{d}_{k}=d_{k}(\check{\mathbf{p}})$ and $\widehat{d}_{k}=d_{k}(\widehat{\mathbf{p}})$. $\check{\mathbf{p}}$ is the optimal solution to $P 2$ if and only if $\check{d}_{1} \leq 0$, and $\widehat{\mathbf{p}}$ is the optimal solution to $P 2$ if and only if $\widehat{d}_{1} \geq 0$.

However, when $\check{d}_{1}>0$ and $\widehat{d}_{1}<0$, whether there exists a EE water-level to make $\check{\mathbf{p}}(x)$ optimal is still not answered. We should study the relation between $\eta_{\mathrm{EE}}^{(\rho)}(\mathbf{p})$ and the EE waterlevel $x$.
3.3. Analytical Expression of $\eta_{E E}^{(\rho)}(x)$. According to (8), $\check{\mathbf{p}}(x)$ is a piecewise function and $1 / \gamma_{n}$ is its discontinuity point. If we sort all discontinuity points in an ascending order such that $1 / \gamma_{1} \leq \cdots \leq 1 / \gamma_{N}$, the interval $\left(1 / \gamma_{1}, \infty\right)$ can be divided into $N$ subintervals, that is, $\left(1 / \gamma_{1}, 1 / \gamma_{2}\right], \ldots,\left(1 / \gamma_{N}, 1 / \gamma_{N+1}\right)$ with $1 / \gamma_{N+1}=+\infty$. Moreover, $\check{\mathbf{p}}(x)$ is continuous when $x \in\left(1 / \gamma_{c}, 1 / \gamma_{c+1}\right], \forall c \in[1, N]\left(\left(1 / \gamma_{c}, 1 / \gamma_{c+1}\right]\right.$ is named as the continuous power interval hereafter). To simplify our analysis and get the closed-form $\eta_{\mathrm{EE}}^{(\boldsymbol{\rho})}(\check{\mathbf{p}}(x))$, we further assume that the water-level rise interval is $\left(\check{x}_{1}, \check{x}_{1+1}\right]$, and the continuous power interval is $\left(1 / \gamma_{c}, 1 / \gamma_{c+1}\right] \subseteq\left(\check{x}_{l}, \check{x}_{l+1}\right]$. Then, according to the definition of the subcarrier subset, we have $\mathcal{N}_{1}=\{n \mid$ $\left.x>1 / \gamma_{n}, k(n) \in \mathscr{K}_{1}\right\}$, and let $M=\left|\mathscr{N}_{1}\right|$. In this case, $\eta_{\mathrm{EE}}^{(\rho)}(\check{\mathbf{p}}(x))$ can be transformed into a continuous function of the water-level $x$; that is

$$
\eta_{\mathrm{EE}}^{(\rho)}(x) \triangleq \frac{\left(B M \log _{2} x+B r_{0}\right)}{\left(\zeta M x+p_{0}\right)},
$$

where

$$
\begin{gathered}
p_{0} \triangleq P_{C}-\zeta \sum_{n=1}^{M} \frac{1}{\gamma_{n}}+\zeta \sum_{n \in \mathcal{N}_{2}} \check{p}_{n}, \\
r_{0} \triangleq \sum_{n=1}^{M} \log _{2} \gamma_{n}+\sum_{k \in \mathscr{K}_{2}} \frac{R_{k}}{B} .
\end{gathered}
$$

The domain of $\eta_{\mathrm{EE}}^{(\rho)}(x)$ is $\mathscr{D}_{l}=\left\{x \in\left(1 / \gamma_{c}, 1 / \gamma_{c+1}\right] \subseteq\left(\check{x}_{l}, \check{x}_{l+1}\right]\right\}$. It is noteworthy that $p_{0}$ and $r_{0}$ are constant as long as the water-level rise interval and continuous power interval are determined.

Similar to $\eta_{\mathrm{EE}}^{(\boldsymbol{\rho})}(\mathbf{p}), \eta_{\mathrm{EE}}^{(\boldsymbol{\rho})}(x)$ is also strictly pseudo-concave, and the first-order derivative is

$$
\frac{d \eta_{\mathrm{EE}}^{(\rho)}(x)}{d x}=\frac{M}{\left(\zeta M x+p_{0}\right)} \cdot f(x),
$$

where

$$
f(x) \triangleq \frac{B}{(\ln 2 \cdot x)}-\zeta \eta_{\mathrm{EE}}^{(\rho)}(x) .
$$

According to the first-order optimality condition, a stationary point $x_{0}$ of $\eta_{\mathrm{EE}}^{(\rho)}(x)$ is the root of the equation $f(x)=0$. The closed-form expression of $x_{0}$ is given by Theorem 3 .

Theorem 3. If there exists a stationary point $x_{0}$ in the domain of $\eta_{E E}^{(\rho)}(x)$, its closed-form expression is given by

$$
x_{0}=\frac{p_{0} / \zeta M}{W\left(\left(p_{0} / \zeta M\right) \cdot 2^{r_{0} / M} e^{-1}\right)},
$$

where $W(\cdot)$ represents the Lambert-W function.

The proof of the theorem can be found in [11].

3.4. EE-Optimal Water-Level. According to the strict pseudoconcavity, $\eta_{\mathrm{EE}}^{(\rho)}(x)$ is maximized at the stationary point $x_{0}$. However, whether the corresponding $\check{\mathbf{p}}\left(x_{0}\right)$ is the global optimal solution to $\mathrm{P} 2$ still needs to be verified. 
Theorem 4. If $x_{0} \in \mathscr{D}_{l}$ is the stationary point of $\eta_{E E}^{(\rho)}(x)$ given by (21), then $\check{\mathbf{p}}\left(x_{0}\right)$ is the global optimal solution to P2.

Proof. Since $x_{0} \in \mathscr{D}_{l}$, it can be verified that $\check{\mathbf{p}}\left(x_{0}\right)$ is a feasible solution to P2. Besides, because $x_{0}$ is a stationary point of $\eta_{\mathrm{EE}}^{(\boldsymbol{\rho})}(x)$, we have $\partial \eta_{\mathrm{EE}}^{(\boldsymbol{\rho})}\left(\check{\mathbf{p}}\left(x_{0}\right)\right) / \partial p_{n}=0\left(n \in \mathcal{N}_{1}\right)$. According to Property 1 , we can show that $\partial \eta_{\mathrm{EE}}^{(\boldsymbol{\rho})}\left(\check{\mathbf{p}}\left(x_{0}\right)\right) / \partial p_{n} \leq$ $0\left(n \in \mathcal{N}_{0}\right.$ or $\left.\mathcal{N}_{2}\right)$. Then it can be verified that $\check{\mathbf{p}}\left(x_{0}\right)$ satisfies the KKT conditions. Hence $\check{\mathbf{p}}\left(x_{0}\right)$ is the optimal solution to P2.

In addition, the existence of $x_{0}$ is proved by Theorem 5 .

Theorem 5. If neither $\check{\mathbf{p}}$ nor $\widehat{\mathbf{p}}$ is the optimal solution to $\mathrm{P} 2$, there must exist $x_{0} \in(\check{x}, \widehat{x})$ such that $\check{\mathbf{p}}\left(x_{0}\right)$ is the optimal solution to $P 2$.

Proof. According to the intermediate value theorem, to prove Theorem 5, we should show that there must exist a continuous power interval $(a, b]$ such that $f(a)>0, f(b) \leq 0$.

In fact, if neither $\check{\mathbf{p}}$ nor $\widehat{\mathbf{p}}$ is the optimal solution to $\mathrm{P} 2$, we can verify that $f(\check{x})>0$ and $f(\widehat{x})<0$ according to Property 1 . Assume $(\check{x}, \widehat{x})$ is divided into $L$ water-level rise intervals. It can be proved that there must exist a water-level rise interval $(c, d]$ such that $f(c)>0, f(d) \leq 0$. If there does not exist such an interval, it can be deduced that $f(\check{x}) \cdot f(\widehat{x})>0$, which yields a contradiction.

Furthermore, assume that there are $N_{1}$ discontinuity points in $(c, d]$ such that $c \leq 1 / \gamma_{n} \leq \cdots \leq 1 / \gamma_{n+N_{1}} \leq d$. Similarly, there must exist an interval $(a, b]$ such that $f(a)>$ $0, f(b) \leq 0$ among $N_{1}+1$ continuous power intervals. Hence, there must be a $x_{0} \in(\check{x}, \widehat{x})$ satisfying $f\left(x_{0}\right)=0$. Based on Theorem $4, \check{\mathbf{p}}\left(x_{0}\right)$ is the optimal solution to P2.

\section{Optimal Energy-Efficient Subcarrier Assignment}

In this section, we will provide a sufficient condition for the optimal energy-efficient subcarrier assignment (ESA) based on the relation between EE and the total transmitting power $P_{T}$. By utilizing this sufficient condition, a quick search method can be devised to obtain the optimal ESA, which will be described in the next section.

According to (4a)-(4e), a feasible ESA can be obtained by solving a rate adaptive (RA) problem for a given total transmitting power $P_{T}$. Moreover, the maximum EE can only be achieved at one of three different total transmitting powers, including two boundary points $\left(P_{\min }\right.$ and $\left.P_{\max }\right)$ and a stationary point $\bar{P}_{T}$ of the perturbation function of P1 [5]. To obtain the optimal ESA, it should first determine $\bar{P}_{T}$, which is an unknown value. Unfortunately, $\bar{P}_{T}$ is difficult to be determined and only an approximation can be found by the iterative algorithms [5]. Therefore, only the suboptimal ESA can be obtained according to the approximate $\bar{P}_{T}$.

On the other hand, based on the EE water-filling structure discussed in the previous section, the optimal ESA can be obtained by calculating the exact optimal EE for every feasible subcarrier assignment $\rho$ and then selecting the one with the maximum value. This exhaustive search is prohibitive for large $K$ and $N$ in a practical system. However, combining the EE water-filling framework and the property of the perturbation function of $\mathrm{P} 1$, a sufficient condition for the optimal ESA can be established to greatly simplify the search.

Define $\eta_{\mathrm{EE}}\left(P_{T}\right) \triangleq R_{\max }\left(P_{T}\right) /\left(\zeta P_{T}+P_{C}\right)$ as the perturbation function of $\mathrm{P} 1$, where $R_{\max }\left(P_{T}\right)$ represents the maximum overall data rate of the rate adaptive (RA) problem [10] with user data requirements for a given total transmitting power $P_{T}$. Then, we have the following.

Theorem 6. For a feasible subcarrier assignment $\overline{\boldsymbol{\rho}}$, the optimal-EE water-level $x_{0}$ and the maximum EE $\eta_{E E}^{(\bar{\rho})}\left(x_{0}\right)$ are given by (21) and (17), respectively. Correspondingly, the total transmitting power $\bar{P}_{T}\left(x_{0}\right)=\mathbf{1}^{T} \cdot \check{\mathbf{p}}\left(x_{0}\right)$ and the overall data rate $R^{(\overline{\boldsymbol{\rho}})}\left(\bar{P}_{T}\left(x_{0}\right)\right)=\eta_{E E}^{(\overline{\boldsymbol{\rho}})} \cdot\left(\zeta \bar{P}_{T}\left(x_{0}\right)+P_{C}\right)$. If $P_{\min }<\bar{P}_{T}<P_{\max }$ and $R_{\max }\left(\bar{P}_{T}\left(x_{0}\right)\right)=R^{(\overline{\boldsymbol{\rho}})}\left(\bar{P}_{T}\left(x_{0}\right)\right), \overline{\boldsymbol{\rho}}$ is the optimal ESA of P1.

Proof. To prove Theorem 6, we should show $\bar{P}_{T}\left(x_{0}\right)$ derived from the optimal EPA for the fixed subcarrier assignment $\overline{\boldsymbol{\rho}}$ is a stationary point of $\eta_{\mathrm{EE}}\left(P_{T}\right)$. In this case, consider the derivative of $\eta_{\mathrm{EE}}\left(P_{T}\right)$

$$
\frac{d \eta_{\mathrm{EE}}\left(P_{T}\right)}{d P_{T}}=\frac{d R_{\max }\left(P_{T}\right) / d P_{T}-\zeta \eta_{\mathrm{EE}}\left(P_{T}\right)}{\zeta P_{T}+P_{C}} .
$$

If $R_{\max }\left(\bar{P}_{T}\left(x_{0}\right)\right)=R^{(\bar{\rho})}\left(\bar{P}_{T}\left(x_{0}\right)\right)$, we can get $\eta_{\mathrm{EE}}\left(\bar{P}_{T}\left(x_{0}\right)\right)=$ $R^{(\bar{\rho})}\left(\bar{P}_{T}\left(x_{0}\right)\right) /\left(\zeta \bar{P}_{T}\left(x_{0}\right)+P_{C}\right)=\eta_{\mathrm{EE}}^{(\bar{\rho})}\left(x_{0}\right)$. Since $x_{0}$ is the stationary point of $\eta_{\mathrm{EE}}^{(\overline{\boldsymbol{\rho}})}(x)$, it has that $\eta_{\mathrm{EE}}^{(\overline{\boldsymbol{\rho}})}\left(x_{0}\right)=B /\left(\zeta \cdot \ln 2 \cdot x_{0}\right)$ based on (20). On the other hand, $d R_{\max }\left(\bar{P}_{T}\left(x_{0}\right)\right) / d P_{T}=$ $B /\left(\ln 2 \cdot x_{0}\right)[11]$. Then, $d \eta_{\mathrm{EE}}\left(\bar{P}_{T}\left(x_{0}\right)\right) / d P_{T}=0$; that is, $\bar{P}_{T}\left(x_{0}\right)$ is a stationary point of $\eta_{\mathrm{EE}}\left(P_{T}\right)$. When $P_{\min }<\bar{P}_{T}<P_{\max }$, EE is maximized at the stationary point of $\eta_{\mathrm{EE}}\left(P_{T}\right)$. Therefore, $\overline{\boldsymbol{\rho}}$ is the optimal ESA.

Based on Theorem 6, the following proposition can be easily verified.

Proposition 7. If $R_{\max }\left(\bar{P}_{T}\left(x_{0}\right)\right)>R^{(\bar{\rho})}\left(\bar{P}_{T}\left(x_{0}\right)\right)$, then $\eta_{E E}^{\left(\rho_{e}\right)}>$ $\eta_{E E}^{(\bar{\rho})}$, where $\boldsymbol{\rho}_{e}$ is the subcarrier assignment obtained by solving the RA problem with $P_{T}=\bar{P}_{T}\left(x_{0}\right)$.

\section{Joint Energy-Efficient Resource Allocation Algorithm}

Based on the analysis in the previous sections, we develop an optimal energy-efficient resource allocation algorithm with low complexity to solve P1, named as joint energy-efficient resource allocation (JERA) algorithm. Different from the existing algorithms proposed in [4-6], the JERA algorithm consists of two layers to iteratively perform subcarrier assignment and power allocation so as to achieve the optimal solution. The aim of the outer layer is to find a feasible subcarrier assignment for a given total transmitting power, and the inner layer is in charge of energy-efficient power allocation based on the obtained subcarrier assignment. Based on the EE water-filling framework, the optimal EPA 
Input: $P_{\max _{*}}$ and $R_{k}, \forall k \in \mathscr{K}$

Output: $\eta_{\mathrm{EE}}^{*}, \rho^{*}$ and $\mathbf{p}^{*}$

(1) Obtain $\check{\mathbf{p}}$ and $\check{\boldsymbol{\rho}}$ by solving the MA problem, and then get $d \eta_{\mathrm{EE}}\left(P_{T}\right) /\left.d P_{T}\right|_{P_{T}=P_{\min }}$ by $(22)$, where $P_{\min }=\mathbf{1}^{T} \cdot \check{\mathbf{p}}$;

(2) if $d \eta_{\mathrm{EE}}\left(P_{T}\right) /\left.d P_{T}\right|_{P_{*}=P_{\min }} \leq 0$ then

(3) return $\mathbf{p}^{*}=\check{\mathbf{p}}, \boldsymbol{\rho}^{*}=\check{\boldsymbol{\rho}}$ and $\eta_{\mathrm{EE}}^{*}=\eta_{\mathrm{EE}}\left(P_{\min }\right)$;

(4) else

(5) Obtain $\widehat{\mathbf{p}}$ and $\widehat{\boldsymbol{\rho}}$ by solving the RA problem with the power constraint $P_{\max }$, and get $d \eta_{\mathrm{EE}}\left(P_{T}\right) /\left.d P_{T}\right|_{P_{T}=P_{\max }}$ by (22);

(6) end if

(7) if $d \eta_{\mathrm{EE}}\left(P_{T}\right) /\left.d P_{T}\right|_{P_{T}=P_{\max }} \geq 0$ then

(8) return $\mathbf{p}^{*}=\widehat{\mathbf{p}}, \boldsymbol{\rho}^{*}=\widehat{\boldsymbol{\rho}}$ and $\eta_{\mathrm{EE}}^{*}=\eta_{\mathrm{EE}}\left(P_{\max }\right)$;

(9) else

(10) $\boldsymbol{\rho}_{e} \leftarrow \check{\boldsymbol{\rho}}$;

(11) repeat

(12) $\overline{\boldsymbol{\rho}} \leftarrow \boldsymbol{\rho}_{e}$;

(13) Obtain $\bar{P}_{T}$ by performing the OEPA algorithm (see detail in Algorithm 2) with input $\overline{\boldsymbol{\rho}}$, and calculate $\boldsymbol{\rho}_{e}$ by solving the RA problem with $\bar{P}_{T}$;

(14) until $R_{\max }\left(\bar{P}_{T}\left(x_{0}\right)\right)=R^{(\bar{\rho})}\left(\bar{P}_{T}\left(x_{0}\right)\right)$

(15) return $\mathbf{p}^{*}=\overline{\mathbf{p}}, \boldsymbol{\rho}^{*}=\overline{\boldsymbol{\rho}}$ and $\eta_{\mathrm{EE}}^{*}=\eta_{\mathrm{EE}}\left(\bar{P}_{T}\right)$;

(16) end if

Algorithm 1: Joint energy-efficient resource (JERA) allocation.

can be easily obtained in the inner layer. Meanwhile, the outer layer finds a series of subcarrier assignments such that the optimal EEs increase monotonously.

The JERA algorithm is shown in Algorithm 1. From Line (1) to Line (8), the algorithm first verifies whether the boundary point $\left(P_{\min }\right.$ or $\left.P_{\max }\right)$ is optimal by utilizing the strict quasi-concavity of $\eta_{\mathrm{EE}}\left(P_{T}\right)$ shown in [5]. If neither is optimal, the algorithm will search the optimal ESA and calculate the corresponding EPA, as shown from Line (10) to Line (15). Specifically, starting from a feasible subcarrier assignment $\overline{\boldsymbol{\rho}}$, JERA calculates the optimal EPA and hence a new $P_{T}$, which is further used to find a new subcarrier assignment $\boldsymbol{\rho}_{e}$ by solving a RA problem [10]. This procedure repeats until the sufficient condition in Theorem 6 is satisfied.

It is noteworthy that the sequence of EE generated by the JERA algorithm is monotonously increasing according to Proportion 1. Besides, the optimal EE is upper bounded, so that JERA algorithm must converge to the optimal solution.

The OEPA algorithm in Line (13) is devised to get the exactly optimal power allocation based on the EE waterfilling framework, which is summarized as Algorithm 2. According to Theorems 2 and 5, the OEPA algorithm consists of three phases. In the first phase, the minimum water-levels $\check{x}_{k}$ 's are obtained. If the power vector $\check{\mathbf{p}}$ derived from $\check{x}_{k}$ 's is not optimal, the algorithm proceeds to the second phase, where the maximum water-level $\widehat{x}$ under the total transmitting $P_{\max }$ is found. If the maximum power vector $\widehat{\mathbf{p}}$ with $\widehat{x}$ is optimal, the algorithm returns. Otherwise, it searches sequentially for the optimal water-level $x_{0}$ in the feasible region $(\check{x}, \widehat{x})$ in the last phase. Since the number of the searched waterlevel intervals only depends on that of the users and the subcarriers, the average computational complexity of the OEPA algorithm is $\mathcal{O}(N+K)$ in the worst case, while that of the BPA (bisection-based power adaptation) algorithm [5] is proportional to $1 / \delta^{2}$, where $\delta$ is the convergence tolerance.
More importantly, the value of EE obtained by the OEPA algorithm is the exact optimum, whereas the BPA algorithm can only provide an approximation result.

\section{Performance Evaluation}

In this section, simulation results are given to verify the theoretical analysis and the performance of the proposed algorithms. In our simulation, the number of data subcarriers is set to be 72 and the bandwidth of each subcarrier is $15 \mathrm{kHz}$ [5]. The block Rayleigh fading channel model is considered and the Okumura-Hata path loss model is followed; that is, $P L(d)=137.74+5.22 \log (d)$ in decibels, where $d$ is the distance between transmitter and receiver in kilometers. The standard deviation of shadowing is $7 \mathrm{~dB}$, and the thermal noise spectral density is $-174 \mathrm{dBm} / \mathrm{Hz}$ [4]. The circuit power is $20 \mathrm{~W}$ and the maximum transmitting power is $40 \mathrm{~W}$ for the base station [12]. The drain efficiency of power amplifier is assumed to be $38 \%$ [4]. Each user in the simulation has the same minimum rate requirement of $100 \mathrm{kbps}$.

First, we compare the performance of the OEPA algorithm with the other two algorithms: the BPA algorithm [5] and the MWF (multilevel water filling) algorithm [10] for a fixed subcarrier allocation. Although the MWF algorithm is a classical SE-based scheme rather than a EE-based scheme, it is used as a benchmark to measure the difference in the energy efficiency between the two classes of scheme. In this simulation example, the number of users is set to 30 . The users are uniformly distributed in a circle centered at the BS with a variable radius. The results in Figures 1(a) and 1(b) show that the average EE and the system throughput of all the three algorithms decrease with the channel power gain. This is due to the fact that the average channel-gain-to-noise ratio (CNR) of each user decreases with the increase of the distance between the user and the BS, such that more power 


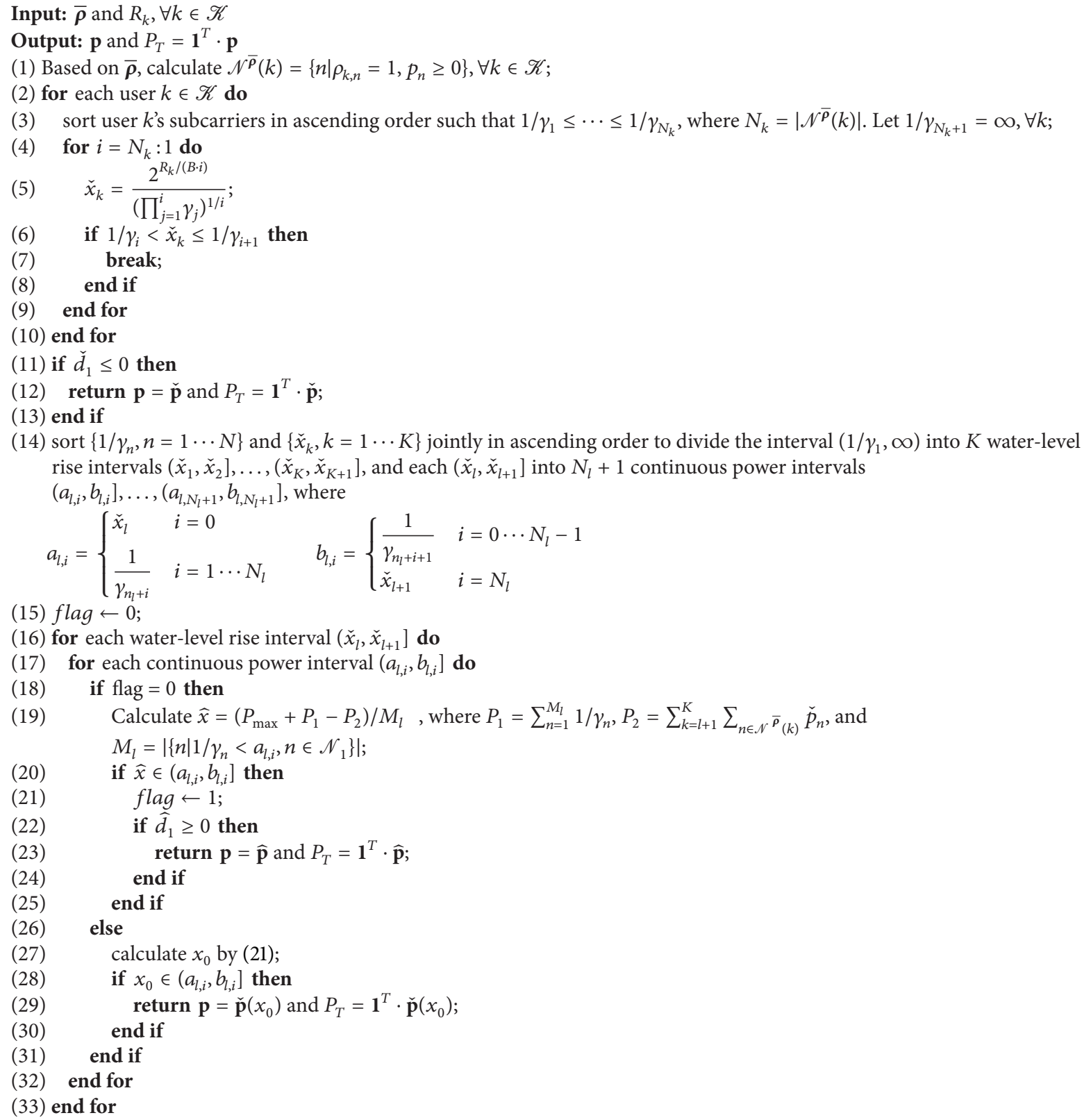

Algorithm 2: Optimal energy-efficient power allocation (OEPA) algorithm.

is required to combat with the severe channel fading to satisfy the data rate constraint of each user. Figure 1(c) illustrates that the SE-based scheme, that is, the MWF algorithm, always depletes all the power to maximize the system throughput. On the contrary, the power consumption of the EE-based scheme, that is, the OEPA algorithm and the BPA algorithm, is adaptive to the channel condition. It is worth noting that there exists a gap between the EE obtained by the OEPA algorithm and the BPA algorithm. The difference is especially $10.15 \mathrm{bit} /$ joule on average when $\delta=10^{-4}$. This stems from the fact that the OEPA algorithm always finds the exact optimal solution, while the BPA algorithm only approaches the optimal value by iterative method. More importantly, the computational complexity of the OEPA algorithm is much lower than that of the BPA algorithm, which is demonstrated in the following simulation example.

In order to compare the computational complexity, assume that there are 10 users evenly distributed in the network and 10000 independent experiments (with different user location) are conducted in the MATLAB environment. Figure 2 shows the CDF of the required CPU time for convergence in different algorithms, where $\delta$ represents the error tolerance of BPA algorithm. From Figure 2, it is obvious that the convergence speed of the OEPA algorithm is superior 


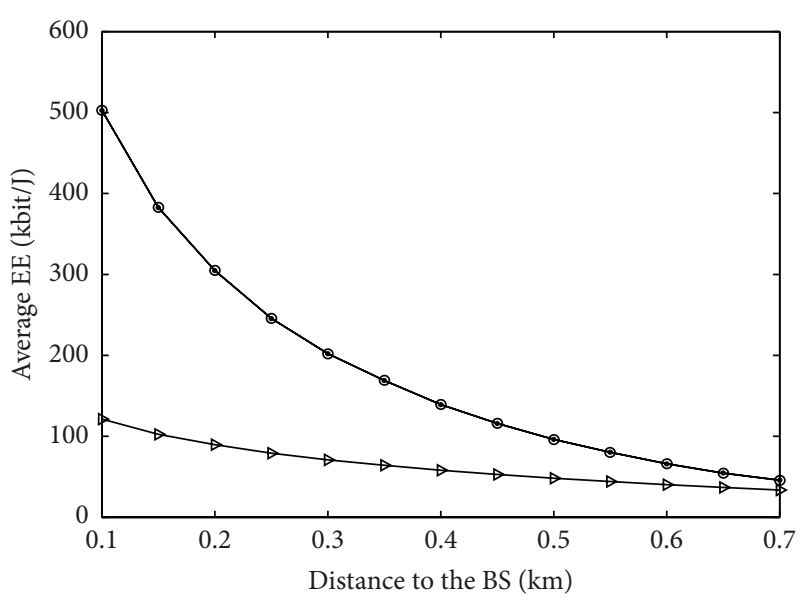

(a) Evaluation and comparison of average $\mathrm{EE}$

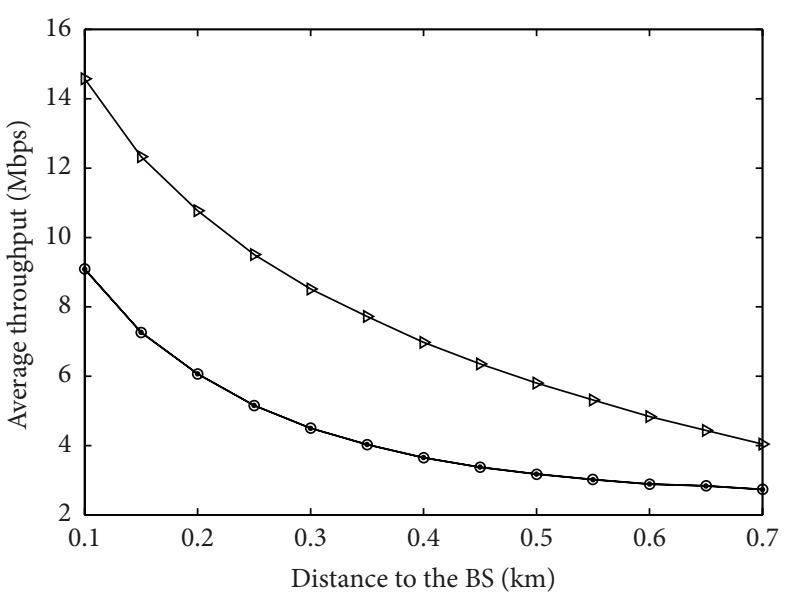

(b) Evaluation and comparison of average throughput

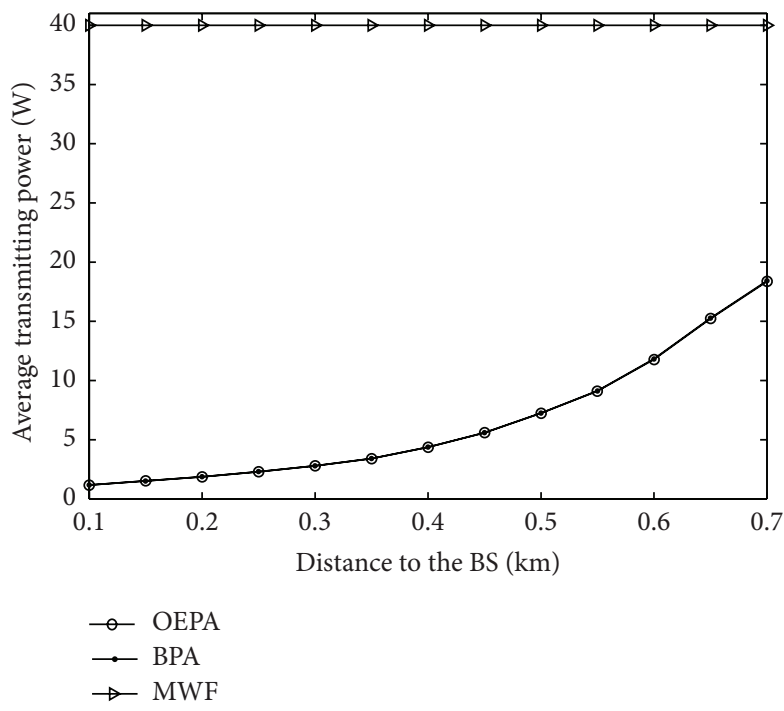

(c) Evaluation and comparison of average transmitting power

FIgURe 1: Performance comparison of different algorithms.

to that of the BPA algorithm. In addition, the complexity of the BPA algorithm increases evidently when the error tolerance becomes tighter. This is due to the fact that the OEPA algorithm can obtain the exact optimal solution by checking at most $K+N$ continuous power intervals in the worst case based on the closed-form expression of EPA. On the contrary, the BPA algorithm is to search bidirectionally for the optimal transmitting power, which results in a higher computational complexity. On average, the CPU time for convergence of the OEPA algorithm is about $15.64 \%$ and $13.03 \%$ of that of the BPA algorithm with $\delta=0.1$ and $\delta=$ 0.001 , respectively.

Moreover, in order to verify the optimality of the proposed JERA algorithm, we compare the EE obtained by JERA with the global optimum obtained by exhaustive search. In this case, we consider a system with 9 subcarriers and 3 users to reduce the complexity of exhaustive search. As shown in Figure 3, the achieved optimal EE in both algorithms decrease with the distance between the BS and users. Moreover, the two curves match with each other very well. It demonstrates the proposed ESA search method based on Theorem 6 is effective and the JERA algorithm can obtain the global optimal ESA and EPA simultaneously.

In addition, we compare the performance of the JERA algorithm with that of the JIOO algorithm [5] with different number of users and subcarriers. The result is shown in Table 1; it can be observed that the EE of the JERA algorithm is superior to that of the JIOO algorithm due to the optimality of the solution obtained by the JERA algorithm. More importantly, the convergence rate of the JERA algorithm is significantly faster than that of the JIOO algorithm. Specifically, the number of iterations for convergence of the JERA algorithm is less than 5 in average, while the JIOO algorithm requires at least 29 iterations in average to approximate the optimum. It is worth noting that each iteration in both algorithms needs to solve a RA problem with rate requirements and total transmitting power constraint. Despite of the nonconvexity of this type RA problem, it has been proven that it can be solved 


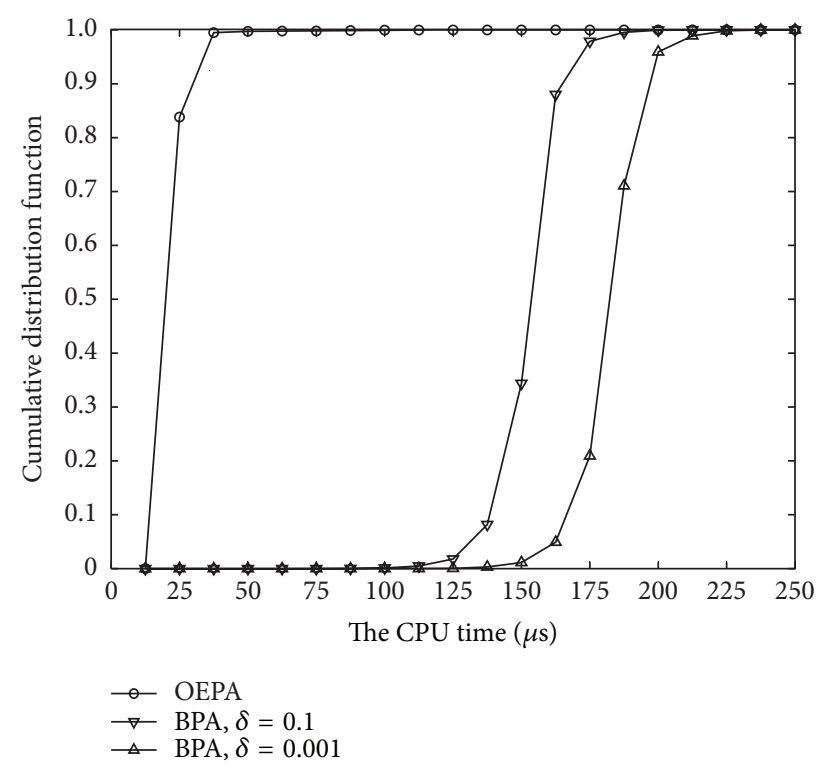

FIGURE 2: Comparison of convergence performance of different algorithms.

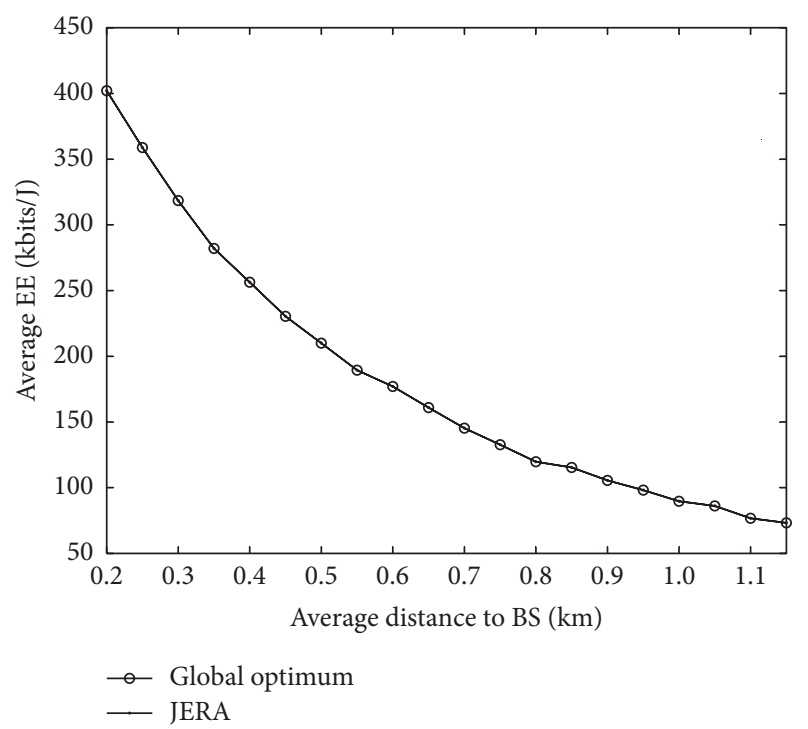

FIGURE 3: Evaluation and comparison of average EE.

efficiently by the Lagrange dual decomposition method with zero duality gap [13].

\section{Conclusion}

In this paper, we investigated the EE maximization problem under both the user rate requirements and the transmitting power constraint. Utilizing the EE water-filling structure, we obtain the closed-form of the optimal EPA. The sufficient condition for optimal ESA is also derived based on the relation between EE and power. Furthermore, we propose a low-complexity algorithm with joint ESA and EPA to address the energy-efficient resource allocation in downlink
TABLE 1: Performance comparison of the two algorithms.

\begin{tabular}{lcccc}
\hline $\begin{array}{l}\text { (Users, } \\
\text { subcarriers) }\end{array}$ & $\begin{array}{c}c \\
\text { Ave. EE } \\
\text { (kbits/Joule) }\end{array}$ & $\begin{array}{c}\text { Ave. } \\
\text { iterations }\end{array}$ & $\begin{array}{c}\text { Ave. EE } \\
\text { (kbits/Joule) }\end{array}$ & $\begin{array}{c}\text { Ave. } \\
\text { iterations }\end{array}$ \\
\hline$(5,16)$ & 81.9 & 2.6 & 81.8 & 29.6 \\
$(10,32)$ & 126.3 & 2.8 & 126.2 & 29.8 \\
$(15,64)$ & 176.6 & 3.2 & 176.5 & 30.1 \\
$(20,64)$ & 179.2 & 3.3 & 179.1 & 30.1 \\
$(25,72)$ & 179.5 & 3.8 & 157.4 & 30.4 \\
$(30,72)$ & 159.8 & 4.3 & 157.6 & 31.7 \\
\hline
\end{tabular}

OFDMA-based networks. Simulation results show that the proposed algorithm achieves the optimal energy-efficient resource allocation with significantly reduced computational complexity compared with the iterative methods.

\section{Conflict of Interests}

The authors declare that there is no conflict of interests regarding the publication of this paper.

\section{Acknowledgments}

This work is supported by the National $\mathrm{S} \& \mathrm{~T}$ Major Project of China under Grant no. 2014ZX03004-003, the Fundamental Research Funds for the Central Universities (no. ZYGX2013J009), China, and EU FP7 Project CLIMBER (PIRSESGA-2012-318939).

\section{References}

[1] D. Feng, C. Jiang, G. Lim, L. J. Cimini Jr., G. Feng, and G. Y. Li, "A survey of energy-efficient wireless communications," IEEE Communications Surveys and Tutorials, vol. 15, no. 1, pp. 167178, 2013.

[2] G. Y. Li, Z. Xu, C. Xiong et al., "Energy-efficient wireless communications: tutorial, survey, and open issues," IEEE Wireless Communications, vol. 18, no. 6, pp. 28-35, 2011.

[3] G. Miao, N. Himayat, and G. Y. Li, "Energy-efficient link adaptation in frequency-selective channels," IEEE Transactions on Communications, vol. 58, no. 2, pp. 545-554, 2010.

[4] C. Xiong, G. Y. Li, S. Zhang, Y. Chen, and S. Xu, "Energy- and spectral-efficiency tradeoff in downlink OFDMA networks," IEEE Transactions on Wireless Communications, vol. 10, no. 11, pp. 3874-3886, 2011.

[5] C. Xiong, G. Y. Li, S. Zhang, Y. Chen, and S. Xu, "Energyefficient resource allocation in OFDMA networks," IEEE Transactions on Communications, vol. 60, no. 12, pp. 3767-3778, 2012.

[6] C. Xiong, G. Y. Li, Y. Liu, Y. Chen, and S. Xu, "Energy-efficient design for downlink OFDMA with delay-sensitive traffic," IEEE Transactions on Wireless Communications, vol. 12, no. 6, pp. 3085-3095, 2013.

[7] S. Cui, A. J. Goldsmith, and A. Bahai, "Energy-efficiency of MIMO and cooperative MIMO techniques in sensor networks," IEEE Journal on Selected Areas in Communications, vol. 22, no. 6, pp. 1089-1098, 2004. 
[8] A. Cambini and L. Martein, Generalized Convexity and Optimization: Theory and Applications, Springer, Berlin, Germany, 2009.

[9] M. S. Bazaraa, H. D. Sherali, and C. M. Shetty, Nonlinear Programming: Theory and Algorithms, Wiley-Interscience, 3rd edition, 2006.

[10] S. Sadr, A. Anpalagan, and K. Raahemifar, "Radio resource allocation algorithms for the downlink of multiuser OFDM communication systems," IEEE Communications Surveys and Tutorials, vol. 11, no. 3, pp. 92-106, 2009.

[11] F. Wu, Y. Mao, X. Huang, and S. Leng, "Low-complexity optimal energy-efficient resource allocation in downlink OFDMA networks," in Proceedings of the International Conference on Computational Problem-Solving (ICCP '12), pp. 46-51, Chengdu, China, October 2012.

[12] Technical Specification Group Radio Access Network, "Further advancements for E-UTRA physical layer aspects (Release 9)," 3GPP Standard TR 36.814 V9.0.0, 2010.

[13] K. Seong, M. Mohseni, and J. M. Cioffi, "Optimal resource allocation for OFDMA downlink systems," in Proceedings of the IEEE International Symposium on Information Theory (ISIT'06), pp. 1394-1398, IEEE, Seattle, Wash, USA, July 2006. 


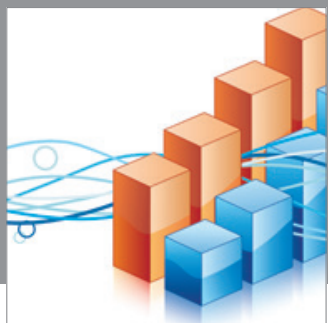

Advances in

Operations Research

mansans

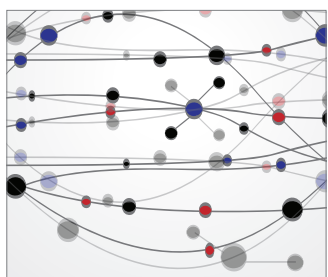

The Scientific World Journal
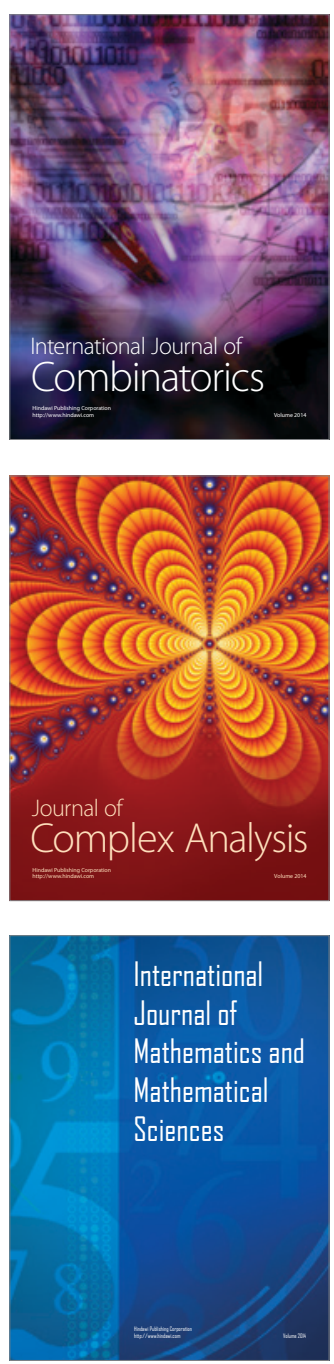
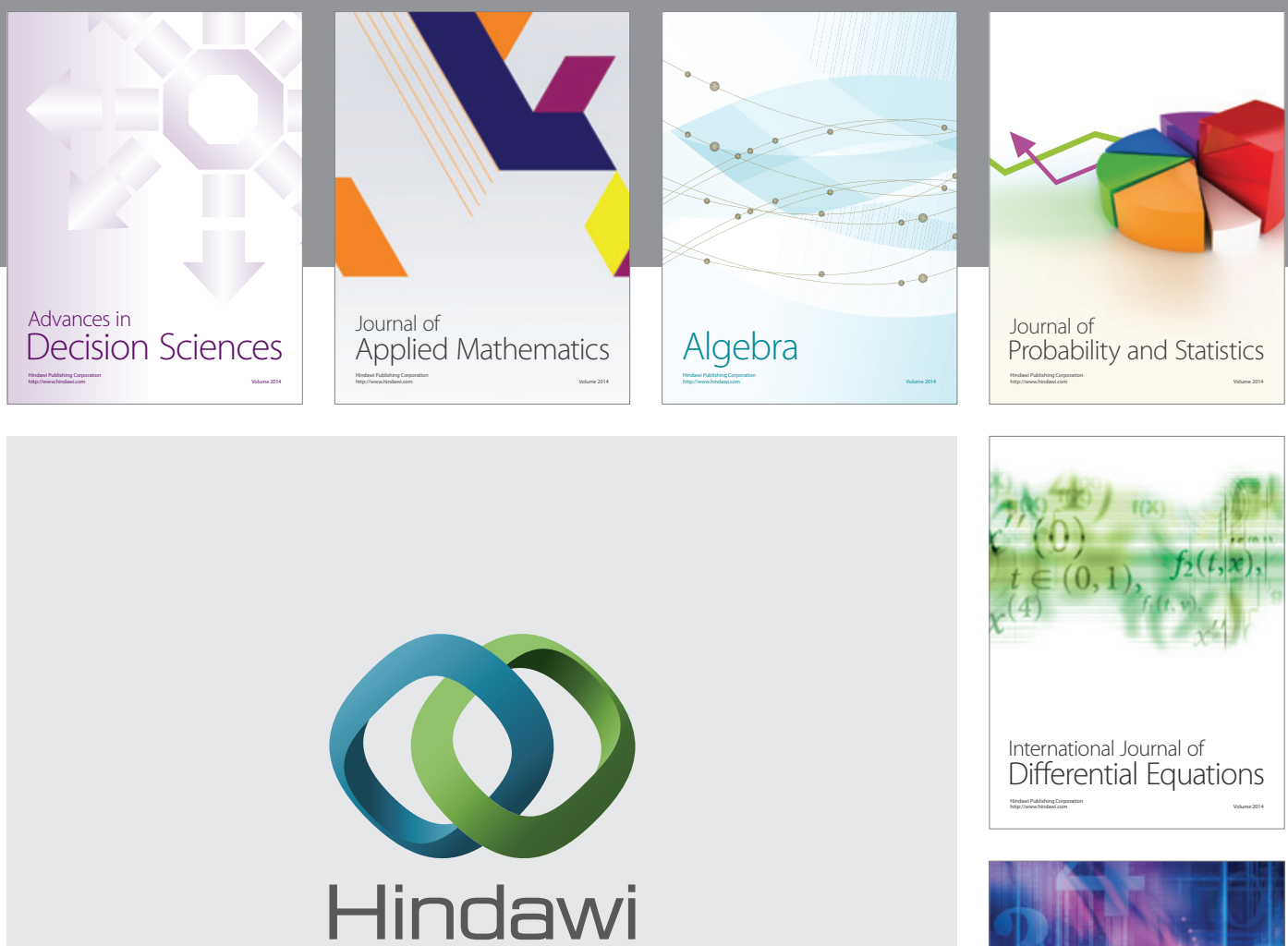

Submit your manuscripts at http://www.hindawi.com
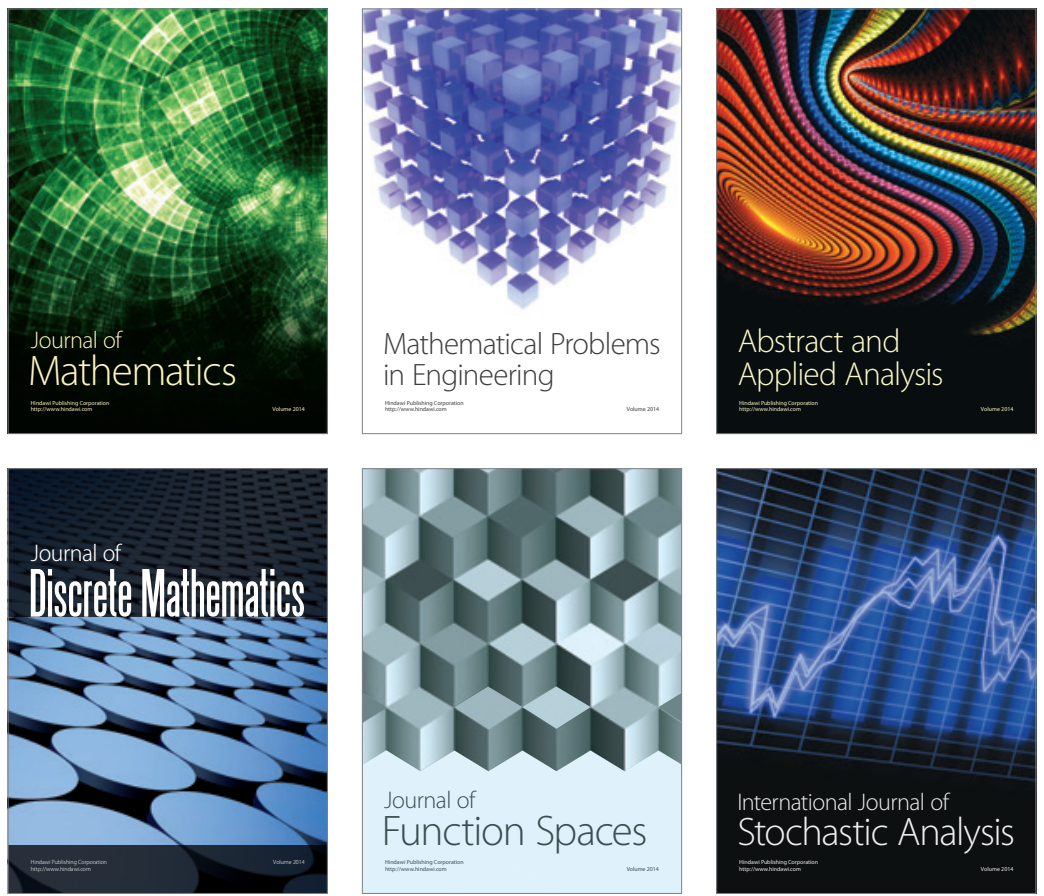

Journal of

Function Spaces

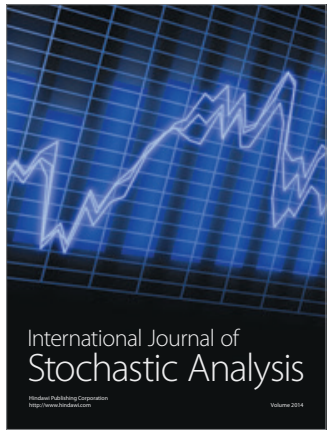

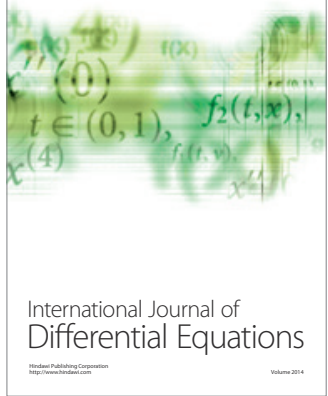
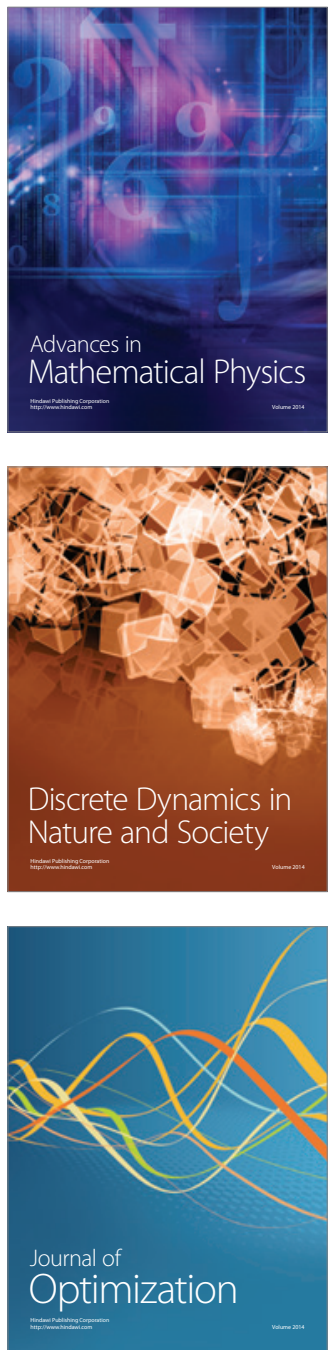